\title{
Adaptive Array Beamforming Using a Chaotic Beamforming Algorithm
}

\author{
Ana Jovanović, Luka Lazović, and Vesna Rubežić \\ Faculty of Electrical Engineering, University of Montenegro, Džordža Vašingtona bb, 81000 Podgorica, Montenegro \\ Correspondence should be addressed to Vesna Rubežić; vesnar@ac.me
}

Received 16 November 2015; Revised 28 January 2016; Accepted 7 February 2016

Academic Editor: Lei Yu

Copyright ( 2016 Ana Jovanović et al. This is an open access article distributed under the Creative Commons Attribution License, which permits unrestricted use, distribution, and reproduction in any medium, provided the original work is properly cited.

\begin{abstract}
The Chaotic beamforming adaptive algorithm is new adaptive method for antenna array's radiation pattern synthesis. This adaptive method based on the optimization of the Least Mean Square algorithm using Chaos theory enables fast adaptation of antenna array radiation pattern, reduction of the noisy reference signal's impact, and the improvement of the tracking capabilities. We performed simulations for linear and circular antenna arrays. We also compared the performances of the used and existing algorithms in terms of the radiation pattern comparison.
\end{abstract}

\section{Introduction}

The successful design of the adaptive antenna system depends on the selection and performance of the beamforming algorithm used for radiation diagram adaptation and adjustment to the specific scenario of incoming signals. Spatial filtering of desired signals and minimizing the impact of interfering signals are necessary in order to achieve improvements of the transmission quality and the wireless communication systems capacity.

The simplest and most widely used adaptive algorithm is the Least Mean Square (LMS) algorithm and its modifications [1-5]. In [2], the authors used an array image factor, sandwiched in between two LMS algorithms sections. They achieve better performance compared to the earlier LMS based algorithms. In recent years the modern optimization and adaptive techniques such as particle swarm optimization [6], sequential quadratic programming [7, 8], Chaos mind evolutionary algorithm [9], genetic algorithm [10], and multiobjective wind driven optimization [11] are used for the antenna arrays radiation diagram's synthesis and modification.

The LMS algorithm and its modifications achieve good results only in cases of antenna arrays with a large number of elements. Also, it is necessary to specify a large number of algorithm parameters with the exactly defined reference signal, which makes these algorithms very complex. This paper uses the Chaotic beamforming adaptive (CBA) algorithm [12] based on the optimization of the LMS algorithm using Chaos theory. The goal of using this algorithm is fast adaptation of the antenna array radiation pattern, the reduction of the noisy reference signal's impact, and the improvement of the tracking capabilities. In the Chaotic beamforming algorithm, the block for Chaotic optimization and algorithm parameters selection is added to the LMS algorithm. The algorithm has been successfully applied to antenna arrays with a different number of antenna elements, with special emphasis on the algorithm performance in the case of antenna arrays with a small number of elements. Criterion for the selection of the used algorithm's optimal parameters is the minimum value of the defined fitness function. Fitness function defines the following requirements: precise estimate of signals' angles of arrival, deep null setting in the diagram of radiation in the direction of the interfering signal, reduction of the main lobe's width, and the level of the side lobes. We compared the performances of the used and existing algorithms in terms of the radiation pattern comparison.

The paper is organized as follows: in Section 2, the antenna arrays theory is presented in brief, in Section 3 the Chaotic beamforming algorithm is described, the simulation results are shown in Section 4, and the concluding comments are given in Section 5. 


\section{Antenna Arrays}

Figure 1 shows the uniform linear and circular antenna arrays formed of $N$ antenna elements, with inter element spacing $d$. The antenna array receives $M$ signals from desired sources $s_{m}(t)$ and the $I$ signals from undesired sources $s_{i}(t)$.

Total signal received by the antenna array $x(t)$ is the sum of the desired signals $x_{m}(t)$, interfering signals $x_{i}(t)$, and white Gaussian noise $n(t)$ :

$$
x(t)=x_{m}(t)+x_{i}(t)+n(t),
$$

where

$$
\begin{aligned}
x_{m}(t) & =A_{M} s_{m}(t), \\
s_{m}(t) & =\left[\begin{array}{llll}
s_{m 1}(t) & s_{m 2}(t) & \cdots & s_{m M}(t)
\end{array}\right]^{T}, \\
x_{i}(t) & =A_{I} s_{i}(t), \\
s_{i}(t) & =\left[\begin{array}{llll}
s_{i 1}(t) & s_{i 2}(t) & \cdots & s_{i I}(t)
\end{array}\right]^{T} .
\end{aligned}
$$

$A_{M}$ and $A_{I}$ are matrices of the desired and interfering signals' "array steering" vectors.

Taking the first antenna in the antenna array as a reference, the matrices are given by the following equations:

$$
\begin{gathered}
A_{M}=\left[\begin{array}{cccc}
1 & 1 & \cdots & 1 \\
e^{j \psi_{1}} & e^{j \psi_{2}} & \cdots & e^{j \psi_{M}} \\
\vdots & \vdots & \cdots & \vdots \\
e^{j(N-1) \psi_{1}} & e^{j(N-1) \psi_{2}} & \cdots & e^{j(N-1) \psi_{M}}
\end{array}\right]_{N \times M}, \\
A_{I}=\left[\begin{array}{cccc}
1 & 1 & \cdots & 1 \\
e^{j \xi_{1}} & e^{j \xi_{2}} & \cdots & e^{j \xi_{I}} \\
\vdots & \vdots & \cdots & \vdots \\
e^{j(N-1) \xi_{1}} & e^{j(N-1) \xi_{2}} & \cdots & e^{j(N-1) \xi_{I}}
\end{array}\right]_{N \times I} .
\end{gathered}
$$

In the case of a linear antenna array, signals from the desired source arrive at the angles $\theta_{1}, \theta_{2}, \ldots, \theta_{M}$, the signals from interfering sources arrive at the angles $\alpha_{1}, \alpha_{2}, \ldots, \alpha_{I}$, and the phase shift of the field between the array's two consecutive elements is given by the following equations:

$$
\begin{gathered}
\psi_{m}=\frac{2 \pi}{\lambda} d \sin \theta_{m}, \quad m=1, \ldots, M, \\
\xi_{i}=\frac{2 \pi}{\lambda} d \sin \alpha_{i}, \quad i=1, \ldots, I .
\end{gathered}
$$

In the case of a circular antenna array at which signals from the desired source arrive at angles $\theta_{1}, \theta_{2}, \ldots, \theta_{M}$ and $\phi_{1}, \phi_{2}, \ldots, \phi_{M}$, and the signals from interfering sources arrive at angles $\alpha_{1}, \alpha_{2}, \ldots, \alpha_{I}$ and $\beta_{1}, \beta_{2}, \ldots, \beta_{I}$, the field phase shift

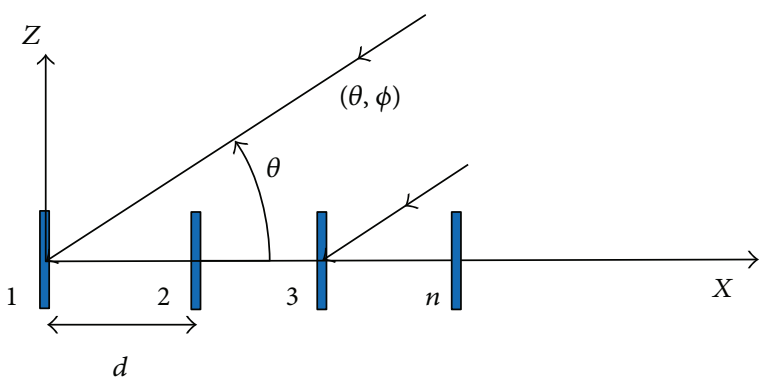

(a)

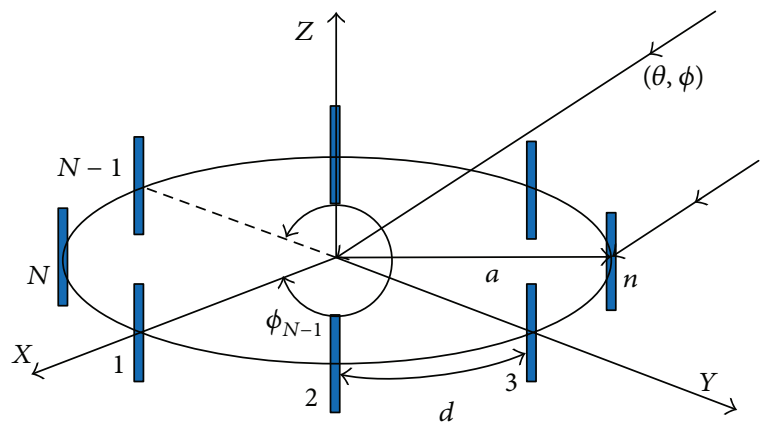

(b)

FIgURE 1: The antenna array geometry: (a) linear and (b) circular.

between two consecutive elements in the array is given by the following equations:

$$
\begin{aligned}
\psi_{m} & =\frac{2 \pi}{\lambda} a \sin \theta_{m} \cos \left(\phi_{m}-\phi_{n}\right), \quad m=1, \ldots, M, \\
\xi_{i} & =\frac{2 \pi}{\lambda} a \sin \alpha_{i} \cos \left(\beta_{i}-\beta_{n}\right), \quad i=1, \ldots, I, \\
\phi_{n} & =\frac{2 \pi}{N}(n-1), \\
\beta_{n} & =\frac{2 \pi}{N}(n-1),
\end{aligned}
$$

The total signal described by relation (1) is applied to the input of the radiation pattern forming network.

\section{Chaotic Beamforming Algorithm}

In this paper, we used the Chaotic beamforming algorithm [12], based on the optimization of the LMS algorithm using Chaos theory. Chaotic systems have several features that make them suitable for use in search procedures and optimization algorithms. These features are great sensitivity to initial conditions, long time unpredictability, and nonrepetition of Chaos [13-15]. That is to say, the chaotic trajectory never visits the same point and that is the main difference between the chaotic search and the search in traditional stochastic optimization techniques. The details about the chaotic search are described in [16]. Different types of chaotic equations that were applied in optimization methods can 


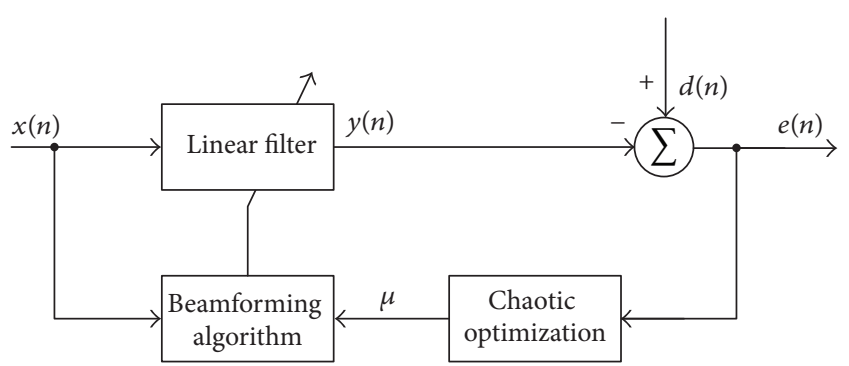

FIGURE 2: Block diagram of the Chaotic beamforming algorithm.

be found in literature [17-19]. In the chaotic beamforming algorithm, we used equations for Chua's oscillators with parameters corresponding to the double-spiral chaotic attractor [13].

Figure 2 shows a block diagram of a chaotic beamforming algorithm. It can be seen that a block for chaotic optimization of step $\mu$ is added to the LMS algorithm.

The task of the chaotic optimization is to determine $X$, which minimizes the fitness function $F(X)$. Vector $X=$ $\left[x_{1}, x_{2}, \ldots, x_{n}\right]$ contains the variables $x_{i} \in\left[L_{i}, U_{i}\right]$, which are limited to the lower $\left(L_{i}\right)$ and upper $\left(U_{i}\right)$ permitted value. In our paper, we adopt $n=1$ and $X=\left[x_{1}\right]=[\mu]$ for the LMS algorithm optimization. The fitness function is defined by the following equation [9]:

$$
\begin{aligned}
F= & k_{1}\left(\frac{\left|\theta_{0}-\theta_{\mathrm{des}}\right|}{180^{\circ}}+\frac{\left|\phi_{0}-\phi_{\mathrm{des}}\right|}{180^{\circ}}\right) \\
& +k_{2} \sum_{i=1}^{N}\left|\mathrm{SLL}_{\mathrm{max}}-\mathrm{SLL}_{\mathrm{des}}\right| \\
& +k_{3}\left(\frac{\theta_{\mathrm{BWFN}}-\theta_{\mathrm{BWFN} \_\mathrm{des}}}{180^{\circ}}+\frac{\phi_{\mathrm{BWFN}}-\phi_{\mathrm{BWFN} \_d e s}}{180^{\circ}}\right) \\
& +k_{4} \sum_{i=1}^{N}\left|\mathrm{NULL}_{\theta_{i} \phi_{i}}-\mathrm{NULL}_{\theta_{i-\mathrm{des}} \phi_{i-\mathrm{des}}}\right|
\end{aligned}
$$

where variables are described as follows.

Description of variables is as follows:

$\theta_{0}, \phi_{0}$ : steering angle of the main lobe.

$\theta_{\text {des }}, \phi_{\text {des }}$ : direction of the desired signal arrival.

$\mathrm{SLL}_{\max }$ : side lobes maximum level.

$\mathrm{SLL}_{\text {des }}$ : side lobes desired maximum level.

$\theta_{\text {BWFN }}:$ main lobe width in the elevation plane.

$\theta_{\text {BWFN_des }}$ : desired main lobe width in the elevation plane.

$\phi_{\mathrm{BWFN}}:$ main lobe width in the azimuth plane.

$\phi_{\text {BWFN_des: }}$ desired main lobe width in the elevation plane.

NULL $_{\theta_{i}}:$ null depth in direction $\theta_{i}$.

NULL $_{\theta_{i_{-} \text {des }}}$ : desired null depth in direction $\theta_{i}$.
We used the following weights in the numerical analysis: $k_{1}=$ $0.3, k_{2}=0.9, k_{3}=0.5$, and $k_{4}=1.9$.

Chaotic optimization is based on the chaotic search. The search procedure which is composed of two parts, global and local search, is shown as follows.

Chaotic search consists of the following:

Global search is as follows:

Step 1. Choosing the parameters for Chua's equations.

Step 2. Initialization of the initial conditions $y_{i}(0)$ for $i=$ $1, \ldots, n$.

Step 3. The normalization of variables $y_{i}$ for $i=1, \ldots, n$ in the range $[0,1]$.

Step 4. The determination of the maximum number of iterations $M_{g}$ for the chaotic global search.

Step 5. Forming variables

$$
x_{i}(k)=L_{i}+z_{i}(k)\left(U_{i}-L_{i}\right), \quad i=1, \ldots, n .
$$

Step 6. In the $k$ th iteration for $X(k)$ fitness function $F(X(k))$ is calculated.

Step 7. The coordinates of the vector $X^{*}$ for which the smallest value of fitness function $F$ was obtained are entered into an algorithm for local search.

Local search is as follows:

Step 1. Determine the number of iterations for local search $M_{L}$.

Step 2. In the $k$ th operation variables $x_{i}(k)=x_{i}^{\bullet} \pm \lambda z_{i}(k)$, $i=1, \ldots, n$ are formed. The sign + or - is selected randomly.

Step 3. Coordinates of vector $X$, for which the lowest value of fitness function $F$ is obtained, are announced for $\mu$ in the LMS algorithm.

\section{Numerical Results}

By using the Chaotic beamforming adaptive algorithm, we obtained linear and circular antenna arrays radiation patterns for different scenarios of incoming signal. We analyzed the impact of noisy reference signal on the algorithm performance in Section 4.1. The influence of the interfering signals' angle of arrival on the algorithm performance is analyzed in Section 4.2. In Section 4.3, the algorithm is applied to antenna arrays with a different number of antenna elements, with special emphasis on the algorithm performance in the case of antenna arrays with a small number of elements.

4.1. The Analysis of the Noisy Reference Signal's Impact on the Algorithm Performance. Here we considered the impact of noisy reference signal on the Chaotic beamforming algorithm performance. White Gaussian noise is added to the reference 


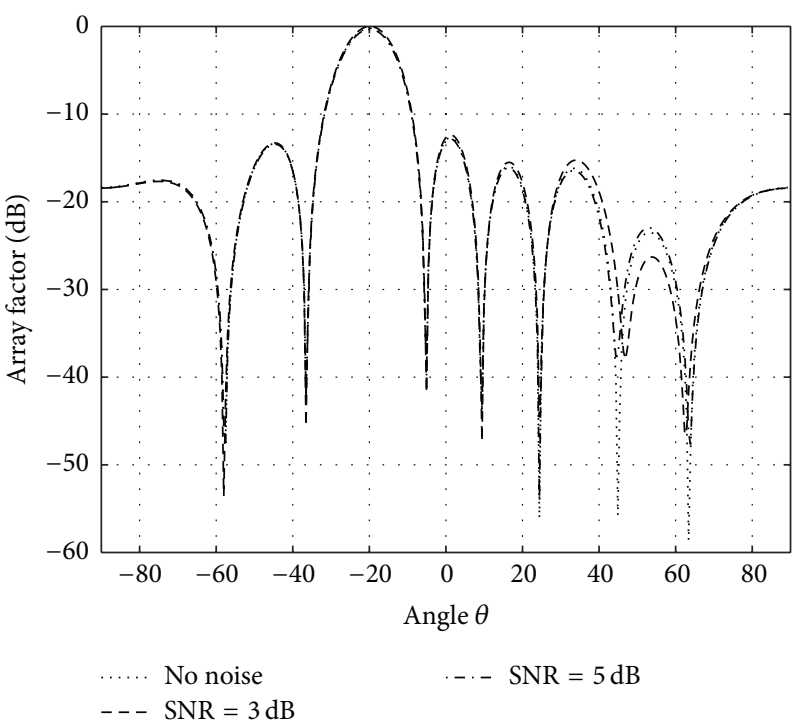

(a)

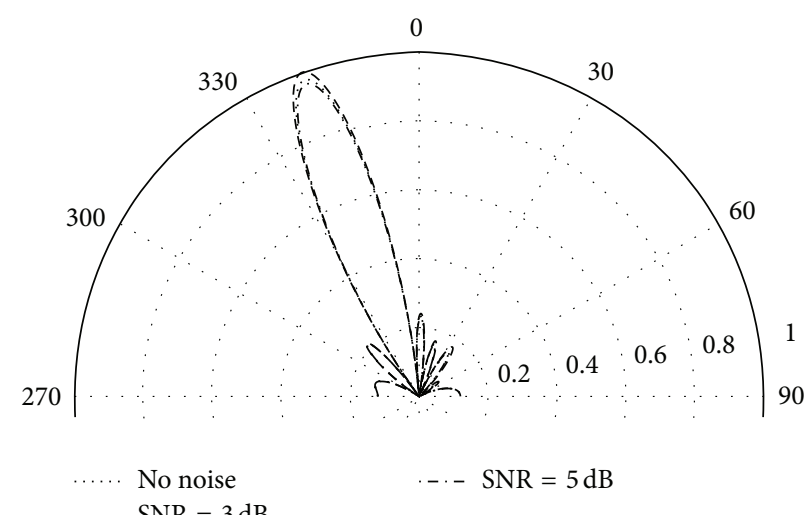

(b)

FIGURE 3: Radiation pattern of normalized array factor for different values of SNR: the angle of arrival of the desired signal is $-20^{\circ}$ and the angle of arrival of the interfering signal is $45^{\circ}$, (a) in the $(x-z)$ plane and (b) in polar coordinates.

signal. Signal to noise ratio (SNR) values used in the simulations were 3,5 , and $7 \mathrm{~dB}$. The obtained radiation patterns for the different SNR values and the combination of the desired and interfering signals' incident angles are shown in a normalized form. The normalization is performed with respect to the diagram with the highest gain in the direction of the desired signal.

Figure 3 shows the normalized radiation diagram obtained when a desired (at an angle $-20^{\circ}$ ) and an interfering signal (at an angle $45^{\circ}$ ) arrive on a linear antenna array. SNR values are 3 and $5 \mathrm{~dB}$. The number of antenna elements is $N=8$ and element spacing is $d=\lambda / 2$.

Figure 4 shows the normalized radiation diagram obtained when a desired (at an angle $-20^{\circ}$ ) and two interfering signals (at angles $-55^{\circ}$ and $45^{\circ}$ ) arrive on a linear antenna array. The value of SNR is $3 \mathrm{~dB}$. The number of antenna elements in the array is $N=8$ and $d=\lambda / 2$.

Based on the results shown in Figures 3 and 4, it can be concluded that the Chaotic beamforming algorithm accurately estimates the desired signal's angle of arrival and sets deep nulls in the directions of the interfering signals for considered levels of noise in the reference signal.

Figure 5 shows the comparative radiation pattern of a linear antenna array obtained using Chaotic beamforming algorithm and LMS algorithm in the cases of accurate and noisy reference signal (the value of the SNR is $3 \mathrm{~dB}$ ). The number of antenna elements of the array is $N=8$ and $d=\lambda / 2$.

Figure 6 shows the comparative diagram of the circular antenna array radiation obtained by Chaotic beamforming algorithm and LMS algorithm in cases of accurate and noisy reference signal (the value of the SNR is $3 \mathrm{~dB}$ ). The number of antenna elements in the array is $N=16$, and the radius of the array is $a=3 \lambda$. Antenna array receives the desired signal

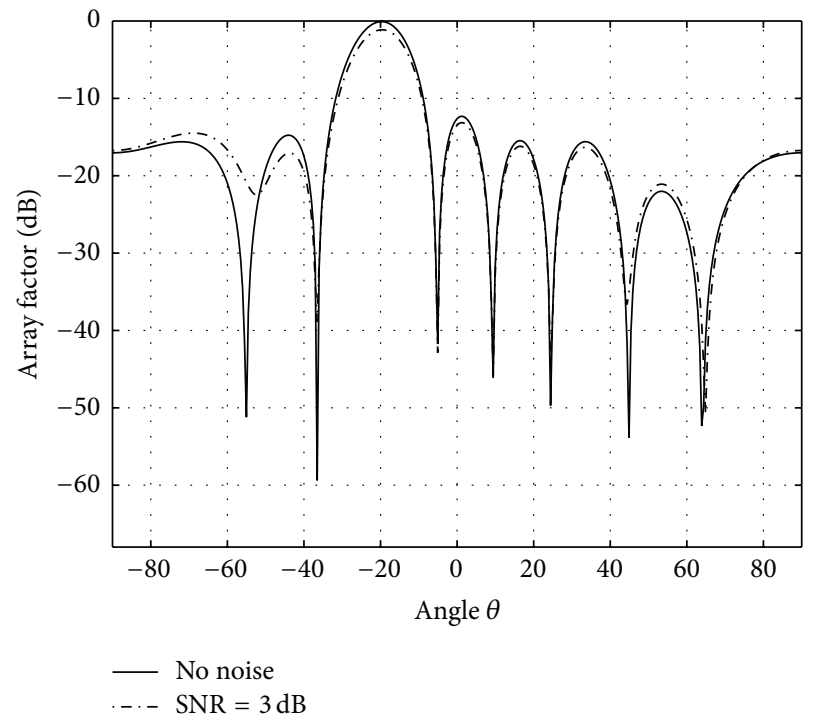

FIGURE 4: Radiation pattern of normalized array factor for different values of SNR: the angle of arrival of the desired signal is $-20^{\circ}$ and the angles of arrival of the interfering signals are $-55^{\circ}$ and $45^{\circ}$.

at an angle $\left(60^{\circ}, 60^{\circ}\right)$ and three interfering signals at angles $\left(60^{\circ}, 90^{\circ}\right),\left(60^{\circ}, 120^{\circ}\right)$, and $\left(60^{\circ}, 240^{\circ}\right)$.

Based on the results shown in Figures 5 and 6, it can be concluded that the Chaotic beamforming algorithm shows robustness to the presence of noise in the reference signal. The Chaotic beamforming algorithm sets deep nulls in the diagram of radiation in the interfering signal's directions of arrival, regardless of the noise level in the reference signal. The LMS algorithm makes an error in estimating the angle of arrival of the interference signal when reference signal is 


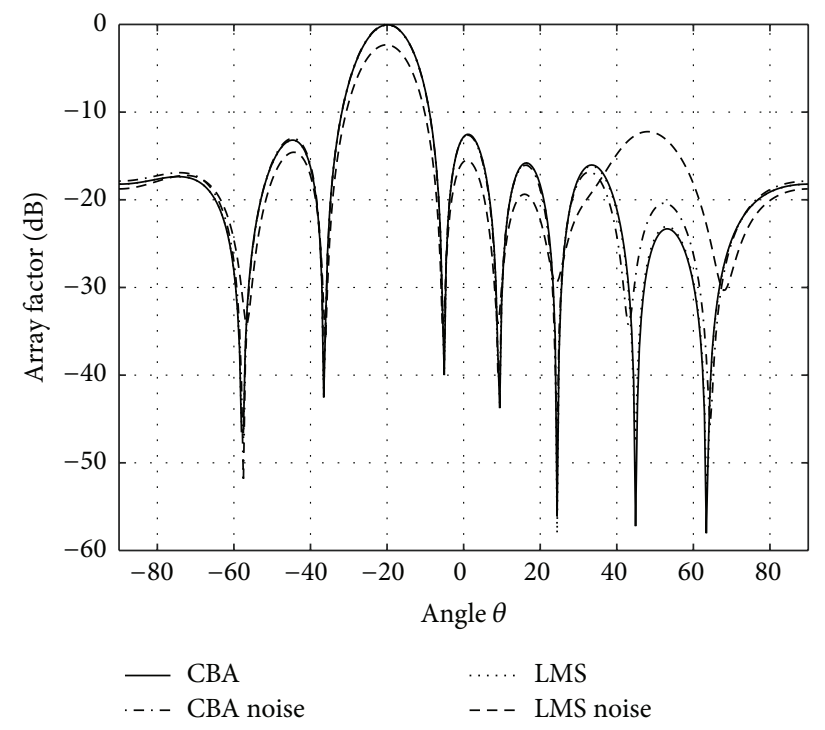

(a)

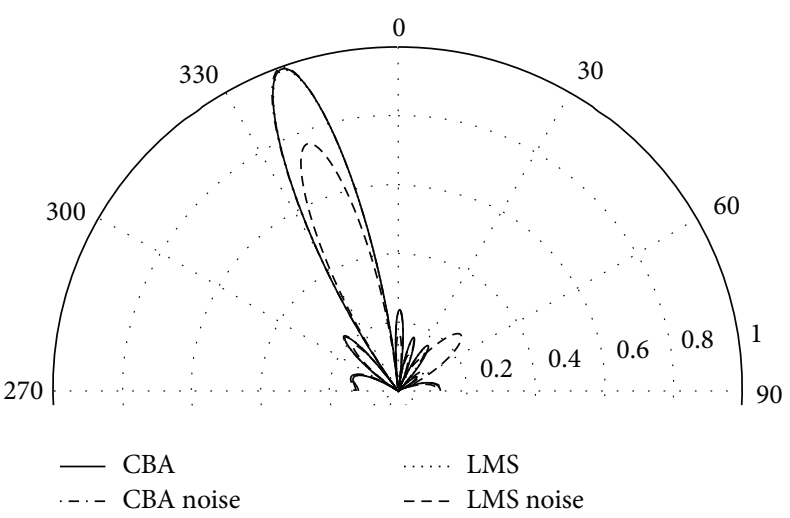

(b)

FIGURE 5: Comparison of the normalized radiation patterns obtained by the Chaotic beamforming algorithm and the LMS algorithm: the angle of arrival of the desired signal is $-20^{\circ}$ and the angle of arrival of the interfering signal is $45^{\circ}$, (a) in the (x-z) plane and (b) in polar coordinates.

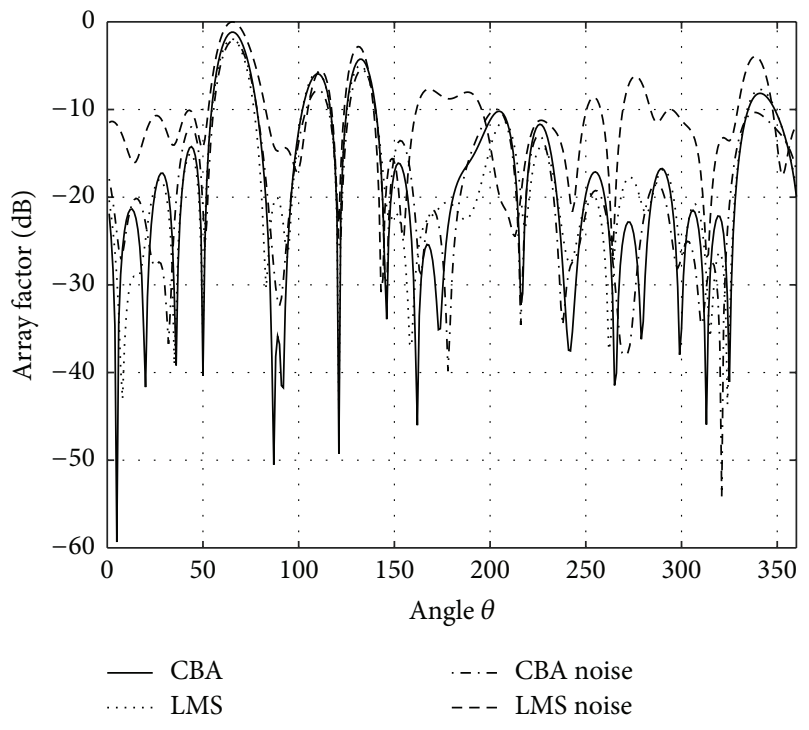

FIGURE 6: Comparison of the circular array radiation patterns obtained by the Chaotic beamforming algorithm and LMS algorithm, shown in plane of azimuthal angle $60^{\circ}$.

noisy. Also in the case of linear array, it has decreased levels of the main lobe in the desired signal's direction.

4.2. The Influence of the Interfering Signal's Angles of Arrival on Algorithm's Performance. Here we considered the effect of interfering signal's angles of arrival on the algorithm performance in cases of accurate and noisy reference signal.
To verify the tracking capabilities of the algorithm, the interfering signal's angles of arrival are selected to be very close to the desired signal's angle of arrival.

The obtained radiation patterns for the different combinations of desired and interfering signal's angles of arrival are shown in a normalized form. In all cases, the number of antenna array elements is $N=10$ and $d=\lambda / 2$.

Figure 7 shows linear arrays radiations patters obtained by the Chaotic beamforming algorithm with noiseless reference signal.

Figure 8 shows the radiation pattern of a linear antenna array obtained by Chaotic beamforming algorithm with noisy reference signal ( $\mathrm{SNR}=7 \mathrm{~dB})$.

Based on the results shown in Figures 7 and 8, it can be concluded that the Chaotic beamforming algorithm accurately estimates the angles of arrival of desired and interfering signals whether the noisy or noiseless reference signal is used. The Chaotic beamforming algorithm sets deep nulls in direction of interfering signals although the interfering signal's angles of arrival are very close to the desired signal's angles of arrival. Based on the foregoing, it can be concluded that the applied algorithm has very good tracking capabilities and suppression of interfering signals.

4.3. The Influence of the Antenna Elements' Number on the Algorithm's Performance. Chaotic beamforming algorithm is applied on antenna arrays with a different number of antenna elements, special emphasis being on the algorithm performance in the case of antenna arrays with a small number of elements. The obtained comparative radiation patterns for different combinations of angles of the desired and interfering signals are shown in a normalized form. Linear antenna arrays with $N=10$ and $N=5$ antenna 


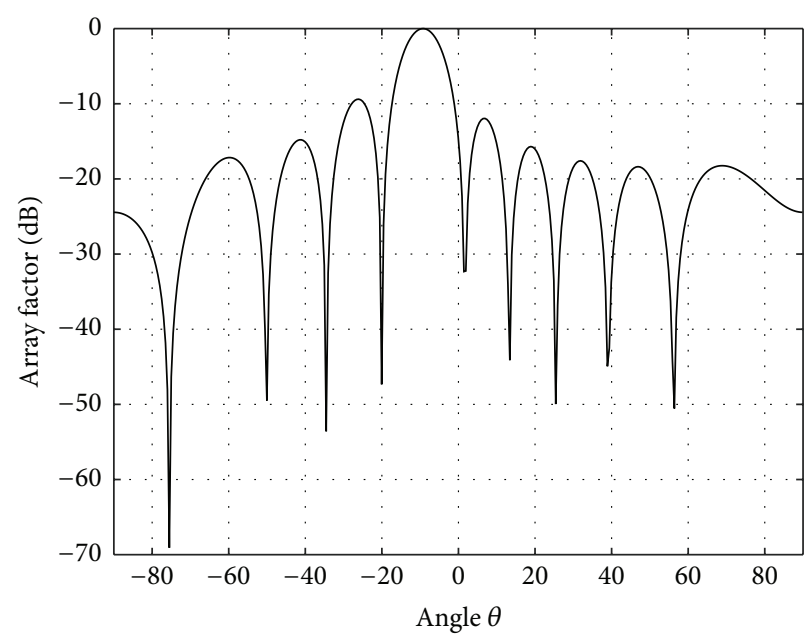

(a)

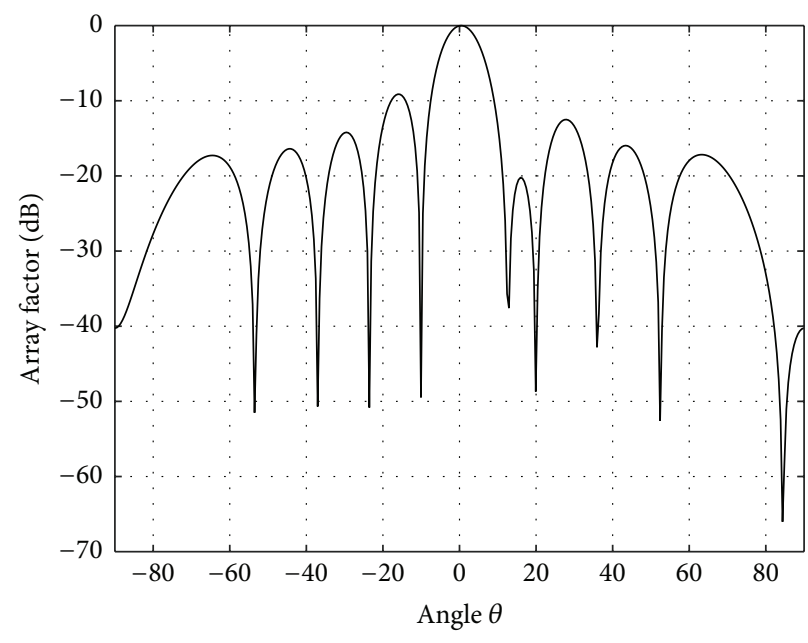

(b)

FIGURE 7: Normalized radiation pattern of the linear antenna array: (a) the angle of arrival of the desired signal is $-10^{\circ}$ and the angle of arrival of the interfering signal is $-20^{\circ}$; (b) the angle of arrival of the desired signal is $0^{\circ}$ and the angles of arrival of the interfering signals are $-10^{\circ}$ and $20^{\circ}$.

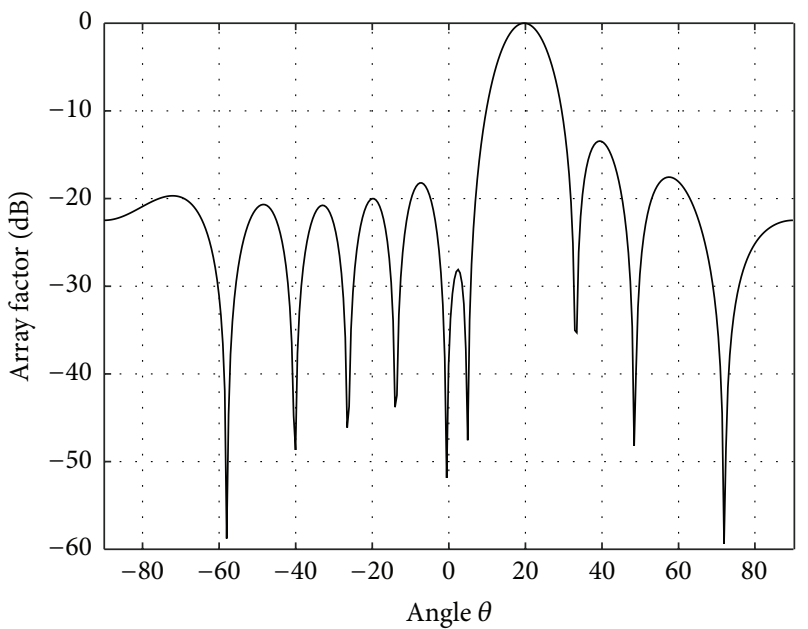

FIGURE 8: Normalized radiation pattern of the linear antenna array: the angle of arrival of the desired signal is $20^{\circ}$ and the angle of arrival of the interfering signal is $5^{\circ}$; $\mathrm{SNR}=7 \mathrm{~dB}$.

elements are analyzed. The distance between the antenna elements is $d=\lambda / 2$, in all cases.

Figure 9 shows the comparative radiation patterns obtained using the Chaotic beamforming algorithm with noiseless reference signal.

Figure 10 shows the comparative antenna arrays radiation pattern obtained by Chaotic beamforming algorithm with noisy reference signal $(\mathrm{SNR}=5 \mathrm{~dB})$.

Based on the results shown in Figures 9 and 10, it can be concluded that the Chaotic beamforming algorithm successfully adjusts radiation pattern on the specific scenario of incoming signals in the case of the antenna array with a small number of elements $(N=5)$. In all the cases, precise estimation angles of arrival of the desired and interfering signals was achieved whether noisy or noiseless reference signal was used. The Chaotic beamforming algorithm sets deep nulls in direction of interfering signals in the case of the antenna array with a small number of elements.

Based on the foregoing, it can be concluded that the applied algorithm performs a very good adaptation of the radiation pattern and suppression of interfering signals in the case of antenna arrays with a small number of elements.

\section{Conclusion}

In this paper, we used the Chaotic beamforming adaptive algorithm, based on the optimization of the LMS algorithm using Chaos theory. The obtained radiation patterns are shown in normalized form, for different values of desired and interfering signals' arrival angles, different levels of noise in the reference signal, and a different number of elements in the array.

We analyzed the influence of the noisy reference signal on the performance of the Chaotic beamforming algorithm. In all analyzed cases, the Chaotic beamforming algorithm accurately directs the main lobe in the desired signal's direction of arrival. It sets deep nulls in the interfering signal's direction of arrival regardless of the reference signal noise level. Based on these results, it can be concluded that the Chaotic beamforming algorithm shows robustness to the presence of noise in the reference signal, which justifies the use of chaotic parameter optimization of LMS algorithms.

To verify the tracking capabilities of the algorithm, interfering signals' angles of arrival are selected to be very close to the desired signal's angles of arrival. The Chaotic beamforming algorithm precisely estimates the interfering signals' direction of arrival and sets deep nulls on the diagram in these directions whether the reference signal is noisy or noiseless. It can be concluded that the applied algorithm has 


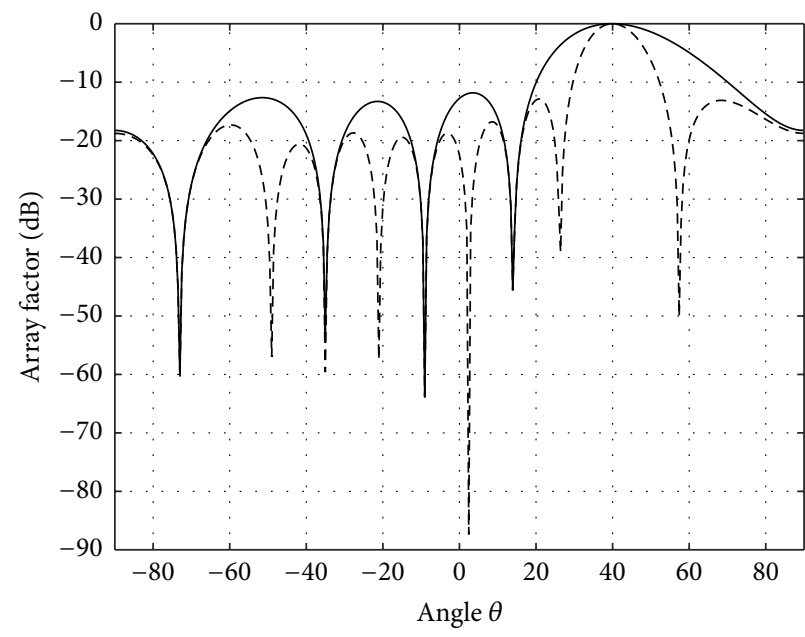

$-N=5$

$--N=10$

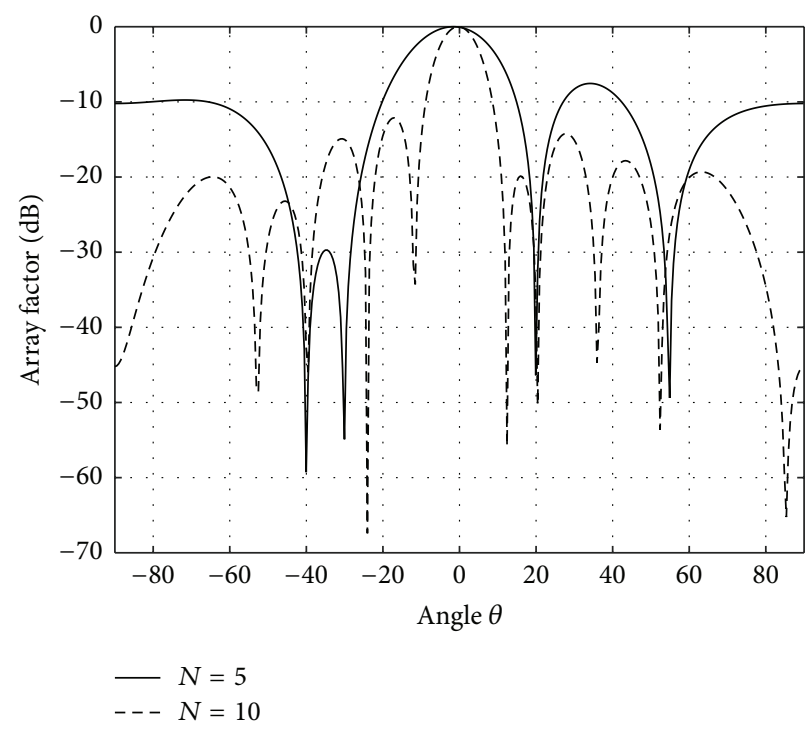

(b)

FIGURE 9: Comparison of the antenna arrays radiation pattern with $N=10$ and $N=5$ elements: (a) the angle of arrival of the desired signal is $40^{\circ}$ and the angle of arrival of the interfering signal is $-35^{\circ}$; (b) the angle of arrival of the desired signal is $0^{\circ}$ and the angles of arrival of the interfering signals are $-40^{\circ}$ and $20^{\circ}$.

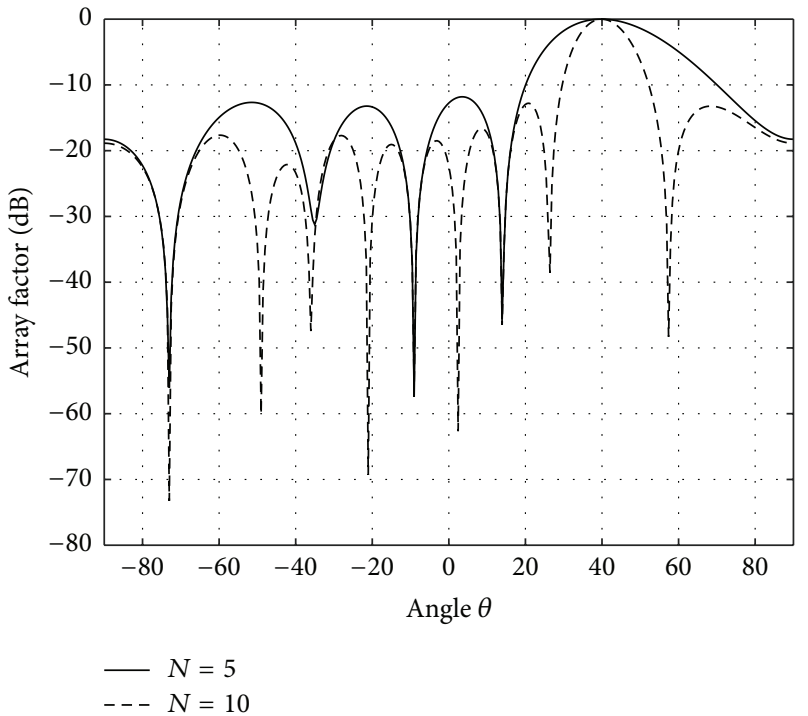

FIGURE 10: Comparison of the antenna arrays radiation pattern with $N=10$ and $N=5$ elements: the angle of arrival of the desired signal is $40^{\circ}$ and the angle of arrival of the interfering signal is $-35^{\circ}$; SNR $=$ $5 \mathrm{~dB}$.

very good tracking capabilities and suppression of interfering signals.

In the cases of antenna array with small number of elements, precise estimation of the desired and interfering signal's angle of arrival is achieved whether noisy or noiseless reference signal was used. In the case of antenna array with a small number of elements, the Chaotic beamforming algorithm sets deep nulls in direction of interfering signals. It can be concluded that the applied algorithm makes very good adjustment of the radiation pattern and suppression of interfering signals in the case of antenna arrays with a small number of elements.

Based on the aforementioned, the Chaotic beamforming algorithm proved to be very precise and relatively simple to use, which makes it suitable it for analysis and adjustment of radiation diagram for complex antenna systems, such as planar and conformal antenna arrays.

\section{Competing Interests}

The authors declare that they have no competing interests.

\section{References}

[1] R. M. Shubair and A. Hakam, "Adaptive beamforming using variable step-size LMS algorithm with novel ULA array configuration," in Proceedings of the 15th IEEE International Conference on Communication Technology (ICCT '13), pp. 650-654, Guilin, China, November 2013.

[2] J. A. Srar, K.-S. Chung, and A. Mansour, "Adaptive array beamforming using a combined LMS-LMS algorithm," IEEE Transactions on Antennas and Propagation, vol. 58, no. 11, pp. 3545-3557, 2010.

[3] R. H. Kwong and E. W. Johnston, "A variable step size LMS algorithm," IEEE Transactions on Signal Processing, vol. 40, no. 7, pp. 1633-1642, 1992.

[4] L. Gargouri, R. Ghayoula, N. Fadlallah, A. Gharsallah, and M. Rammal, "Steering an adaptive antenna array by LMS algorithm," in Proceedings of the 16th IEEE International Conference on Electronics, Circuits, and Systems (ICECS '09), pp. 459-462, Yasmine Hammamet, Tunisia, December 2009.

[5] P. Fletcher and M. Dean, "Low complexity implementation of LMS algorithm," Electronics Letters, vol. 38, no. 15, pp. 836-837, 2002. 
[6] M. M. Khodier and C. G. Christodoulou, "Linear array geometry synthesis with minimum sidelobe level and null control using particle swarm optimization," IEEE Transactions on Antennas and Propagation, vol. 53, no. 8, pp. 2674-2679, 2005.

[7] M. Mouhamadou, P. Armand, P. Vaudon, and M. Rammal, "Interference supression of the linear antenna arrays controlled by phase with use of SQP algorithm," Progress in Electromagnetics Research, vol. 59, pp. 231-249, 2006.

[8] M. Mouhamadou, P. Vaudon, and M. Rammal, "Smart antenna array patterns synthesis: null steering and multi-user beamforming by phase control," Progress in Electromagnetics Research, vol. 60, pp. 95-106, 2006.

[9] J. Liu, N. Li, and K. Xie, "Application of chaos mind evolutionary algorithm in antenna arrays synthesis," Journal of Computers, vol. 5, no. 5, pp. 717-724, 2010.

[10] J. S. Rahhal and D. I. Abu-Al-Nadi, "A general configuration antenna array for multi-user systems with genetic and ant colony optimization," Electromagnetics, vol. 27, no. 7, pp. 413426, 2007.

[11] Z. Zhang, S. Salous, H. Li, and Y. Tian, "An opportunistic array beamforming technique based on binary multiobjective wind driven optimization method," International Journal of Antennas and Propagation, vol. 2015, Article ID 495879, 7 pages, 2015.

[12] L. Lazovic, A. Jovanovic, and V. Rubezic, "Chaos based optimization of LMS algorithm applied on circular antenna arrays," in Proceedings of the 4th Mediterranean Conference on Embedded Computing (MECO '15), pp. 439-442, Budva, Montenegro, June 2015.

[13] M. P. Kennedy, “Three steps to chaos. II. A Chua's circuit primer," IEEE Transactions on Circuits and Systems. I. Fundamental Theory and Applications, vol. 40, no. 10, pp. 657-674, 1993.

[14] J. C. Sprott, Chaos and Time-Series Analysis, Oxford University Press, Oxford, UK, 2003.

[15] V. G. Ivancevic and T. T. Ivancevic, Complex Nonlinearity: Chaos, Phase Transitions, Topology Change, and Path Integrals, Understanding Complex Systems, Springer, Berlin, Germany, 2008.

[16] N. Nedjah, L. dos Santos Coelho, V. C. Mariani, and L. de Macedo Mourelle, "Theory and applications of chaotic optimization methods," in Innovative Computing Methods and Their Applications to Engineering Problems, vol. 357, pp. 131-161, Springer, Berlin, Germany, 2011.

[17] B. Li and W. Jiang, "Optimizing complex functions by chaos search," Cybernetics and Systems, vol. 29, no. 4, pp. 409-419, 1998.

[18] B. Liu, L. Wang, Y.-H. Jin, F. Tang, and D.-X. Huang, "Improved particle swarm optimization combined with chaos," Chaos, Solitons \& Fractals, vol. 25, no. 5, pp. 1261-1271, 2005.

[19] D. Yang, G. Li, and G. Cheng, "On the efficiency of chaos optimization algorithms for global optimization," Chaos, Solitons \& Fractals, vol. 34, no. 4, pp. 1366-1375, 2007. 


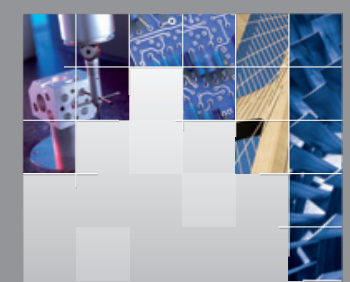

\section{Enfincering}
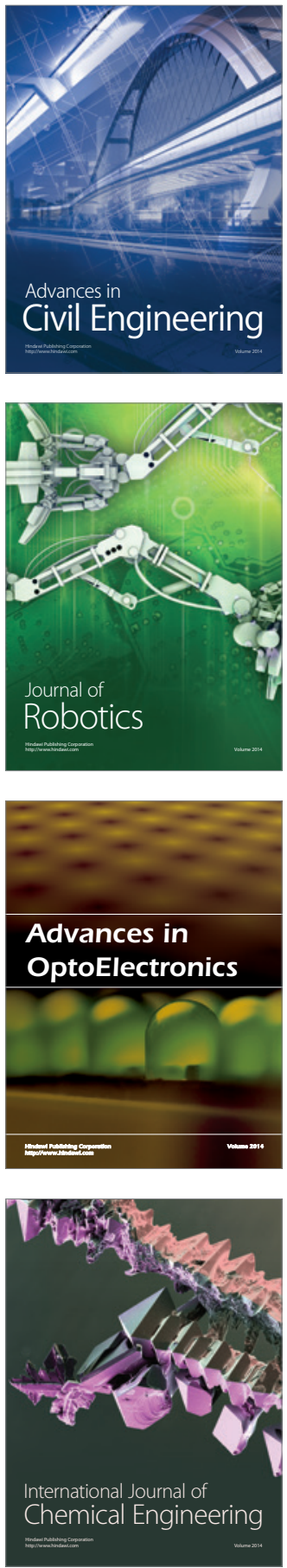

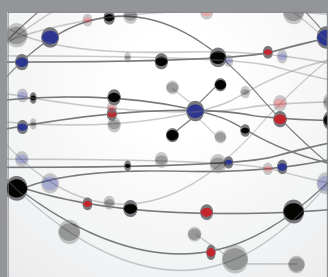

The Scientific World Journal

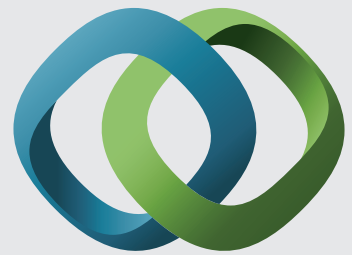

\section{Hindawi}

Submit your manuscripts at

http://www.hindawi.com
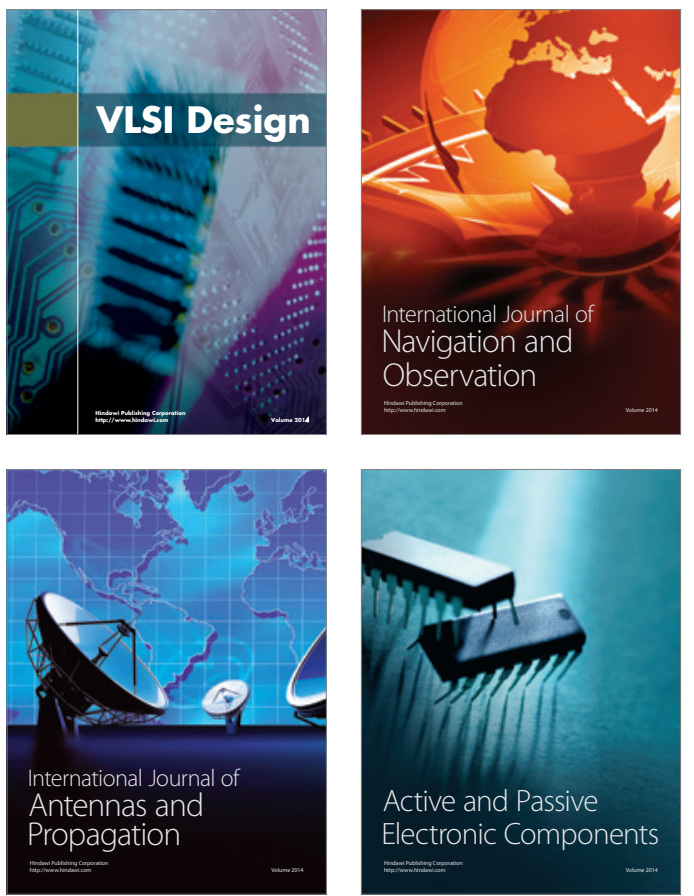
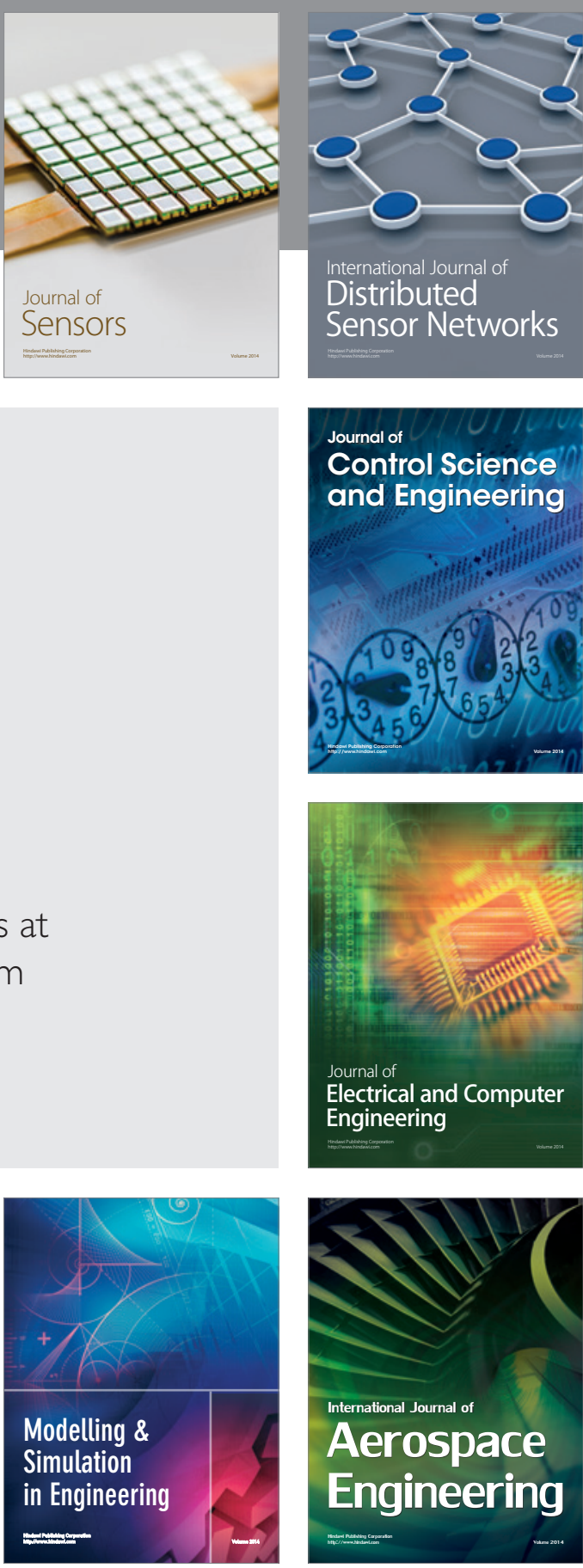

International Journal of

Distributed

Sensor Networks

Journal of

Control Science

and Engineering
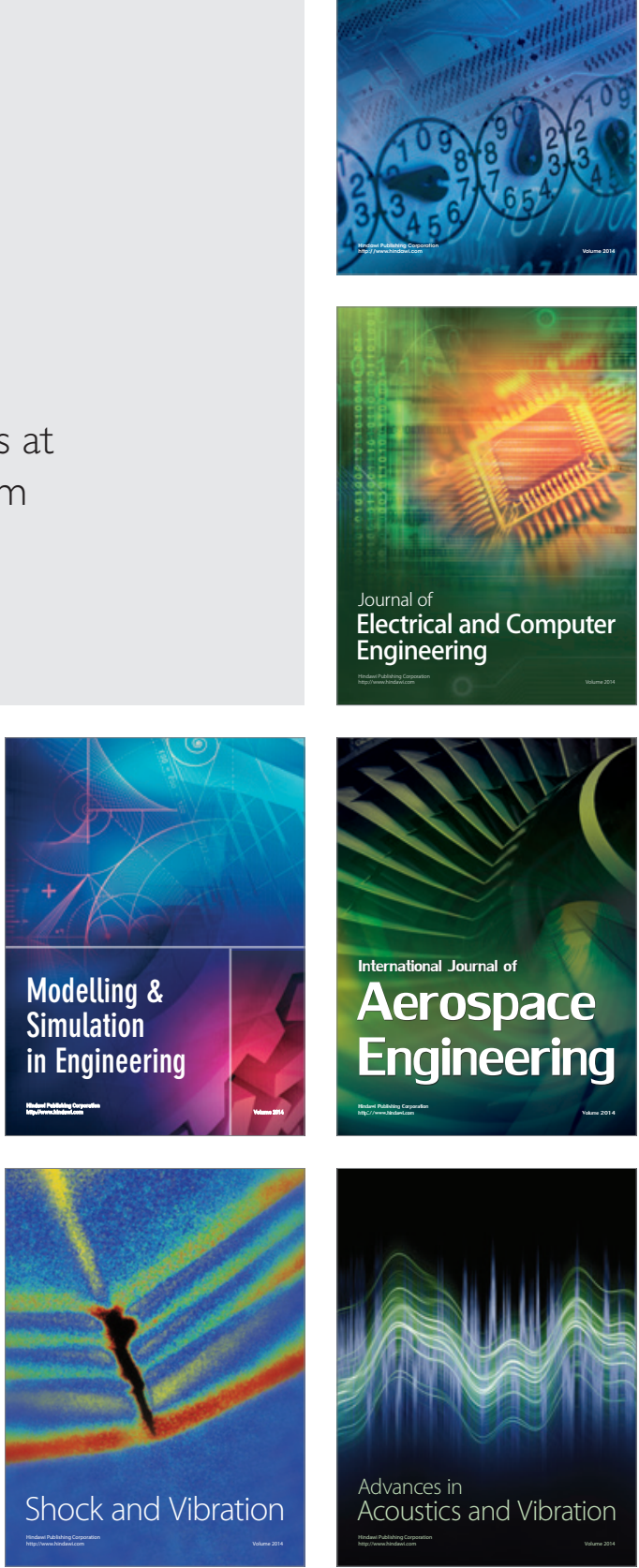